\title{
RELIEF
}

Revue électronique de littérature française

\section{(Re)traduire des classiques français en breton}

Mannaig Thomas, Université de Bretagne occidentale $\square$ Philippe Lagadec, Université de Bretagne occidentale $\square$

RELIEF - Revue électronique de littérature française Vol. 15, $\mathrm{n}^{\circ} 1$ : «(Re)Traduire les classiques français », dir. Maaike Koffeman et Marc Smeets, juillet 2021

ISSN 1873-5045, publié par Radboud University Press Site internet : www.revue-relief.org

Cet article est publié en libre accès sous la licence CC-BY 4.0

\section{Pour citer cet article}

Mannaig Thomas et Philippe Lagadec, «(Re)traduire des classiques français en breton ", RELIEF-Revue électronique de littérature française, vol. $15, n^{\circ} 1$, 2021, p. 114-125. doi.org/10.51777/relief10833 


\title{
(Re)traduire des classiques français en breton
}

\author{
Mannaig Thomas et PhiLIPPe LAGADEC, Centre de recherche bretonne et celtique, \\ Université de Bretagne occidentale
}

\begin{abstract}
Résumé
Dans le cadre d'une politique volontariste d'aide à la publication d'ouvrages en langue bretonne par le Conseil régional de Bretagne, cet article s'intéresse au dispositif d'aide à la (re)traduction en breton d'œuvres du «patrimoine littéraire mondial » et plus particulièrement celles écrites en langue française. Comme ces (re)traductions ne s'expliquent pas par la nécessité de rendre ces œuvres accessibles - le lectorat du breton étant francophone -, c'est donc qu'elles ont d'autres objectifs à replacer dans le contexte plus large de la politique de promotion, de diffusion et d'extension sociale de la langue bretonne.
\end{abstract}

Selon Alain Viala, les classiques sont « plus une invitation à réfléchir sur l'histoire que des illustrations d'une esthétique transcendantale ${ }^{1}$ ». C'est dans cette perspective que nous proposons de nous interroger sur la politique volontariste de traductions littéraires en breton encouragée par le Conseil régional de Bretagne depuis 2013. Ayant pour objectif de faire traduire et publier des « œuvres du patrimoine littéraire mondial ", l'institution régionale a mis en place un dispositif d'aide à la traduction en langue bretonne pour une liste d'œuvres établie par une commission mise en place par l'Office public de la langue bretonne (OPLB) ${ }^{2}$. Dans cette liste figurent, en particulier, un certain nombre d'œuvres en français qui ont plus particulièrement attiré notre attention car elles font l'objet d'un type de traduction particulière : il s'agit d'une forme de (re)traduction, car ce sont des textes que tous les locuteurs du breton (" 225000 locuteurs actifs de plus de 2 ans $^{3}$ » en 2018, tous francophones) sont à même de lire dans la langue originale et dont ils partagent l'arrière-plan culturel, souvent grâce à l'école.

Ces (re)traductions ne s'expliquent donc pas par la nécessité de rendre accessible ces textes, elles ne s'expliquent pas, non plus par un éventuel vieillissement de traductions précédentes en breton - qui sont inexistantes -, mais elles constituent pour les traducteurs, et pour les lecteurs, un exercice de re-traduction, et partant, d'une forme de re-lecture d'œuvres déjà connues. Hormis ponctuellement et par curiosité, il ne viendrait sans doute pas à l'idée d'un francophone de faire le choix de lire Molière ou Jean Giono en anglais, en allemand ou toute

1. Alain Viala, "Qu'est-ce qu'un classique? », Littératures classiques, n 19, 1993, p. 31.

2. L'Office public de la langue bretonne (OPLB) est un établissement public de coopération culturelle qui a pour principales missions la promotion de la langue bretonne et le développement de son emploi dans l'ensemble des domaines d'usage d'une langue.

3. OPLB, «Les chiffres clés de la langue bretonne ", www.fr.brezhoneg.bzh/5-chiffres-cles, consulté le $1^{\text {er }}$ mars 2021. 
autre langue qu'il maîtriserait par ailleurs; de plus, la lecture d'œuvres en langue originale, quand elle est possible, est plutôt valorisée, dès lors, pourquoi proposer à des lecteurs, aussi francophones, des œuvres en breton traduites du français? Nous proposons, pour toutes ces raisons, de considérer ce phénomène comme une forme de (re)traduction puisqu'il s'agit, pour le lectorat concerné, de retrouver, sous une forme nouvelle, une œuvre déjà connue ; déjà connue parce que déjà lue ou faisant, en tant que 'classique', partie de la culture, au moins scolaire, des Français pratiquant la lecture ${ }^{4}$.

Au-delà de cette interrogation initiale, ces (re)traductions poussent aussi à s'interroger sur le choix des 'classiques' sélectionnés, mais également sur ce que ces choix disent de la langue-cible, le breton en l'occurrence, du public-cible, ainsi que des acteurs (individus comme institutions) qui établissent cette sélection et sont engagés dans ces publications. Ces (re)traductions rappellent également que loin d'être neutre et symétrique, la traduction littéraire est aussi la manifestation d'un échange inégal entre des champs littéraires dans un univers fortement hiérarchisé5. Ces (re)traductions doivent donc être resituées dans I'histoire sociolittéraire de la littérature en langue bretonne et dans celle de ses rapports continus avec le champ littéraire français (œuvres, auteurs, genres, institutions littéraires et réception par le lectorat) ${ }^{6}$.

Contrairement à ce qui est cru parfois, y compris par les locuteurs eux-mêmes, la langue bretonne n'a pas accédé récemment à l'écrit, et connaît une pratique littéraire relativement ancienne. Utilisée dans des écrits religieux depuis le $\mathrm{XVI} \mathrm{l}^{\mathrm{e}}$ siècle, souvent sous la forme de la traduction-adaptation à cette époque, la littérature en langue bretonne est, comme toutes les autres, à replacer dans le contexte des conditions sociolittéraires qui ont vu son apparition et son développement, ainsi que dans la situation sociolinguistique du breton et son évolution. Pratiquée par la majorité de la population - essentiellement rurale - comme langue quotidienne jusqu'à la Seconde Guerre mondiale, la pratique du breton s'est fortement réduite depuis. Parallèlement à ce phénomène, la langue fait l'objet d'un fort engagement militant depuis le début du XX $x^{\mathrm{e}}$ siècle environ, visant à la fois à contrer la baisse de la pratique, mais aussi à développer l'usage de la langue dans des domaines qui n'avaient pas, ou très peu, été les siens jusqu'alors : I'enseignement en particulier, avec la mise en place d'un cursus scolaire bilingue breton-français dès 1977, - il y a aujourd'hui près de 20000 élèves dans l'enseignement primaire et secondaire. Ainsi, alors que le nombre de locuteurs a continué, et continue de baisser, et que la majorité des locuteurs ne sont pas lecteurs du breton, une littérature dans cette langue continue d'exister grâce, à la fois, à un fort engagement militant et à des dispositifs d'aides à l'édition spécifiques pour des œuvres publiées en langue bretonne.

4. Armelle Vincent Gérard et Julie Poncet, Les Français et la lecture-2019, CNL-Ipsos, 2019.

5. Pascale Casanova, "Consécration et accumulation de capital littéraire. La traduction comme un échange inégal ", Actes de la recherche en sciences sociales, $\mathrm{n}^{\circ}$ 144, 2002, p. 7.

6. Nelly Blanchard et Mannaig Thomas, "Qu'est-ce qu'une périphérie littéraire? », Des littératures périphériques, Rennes, Presses universitaires de Rennes, 2014, p. 14. 
C'est dans ce contexte que, depuis 2004, plusieurs dispositifs d'aides à la publication d'ouvrages en breton ont été successivement mis en place : tout d'abord, une aide à l'impression d'ouvrages et, depuis 2013, une aide à la traduction dont il sera plus spécifiquement question dans cet article. Ce dernier soutien financier est corrélé à plusieurs critères impliquant I'OPLB : il n'est accordé que pour des ouvrages figurant sur une liste pré-établie et il implique une validation, en amont, de la qualité de la traduction proposée. L'un des buts recherchés est une professionnalisation des traducteurs.trices du breton qui sont rémunérés pour ce faire. Ceci constitue une forme d'exception dans l'espace littéraire en langue bretonne largement pris en charge par des bénévoles, hormis quelques rares salariés pour la gestion des maisons d'édition et le travail d'impression des ouvrages.

Ce dispositif d'aide à la traduction de 'classiques' a été mis en place sur le modèle de programmes qui existent pour d'autres langues en situation minoritaire comme le basque ${ }^{7}$, par exemple. Des dispositifs d'aides existent également pour la promotion de la traduction

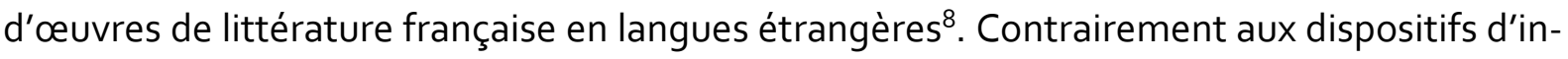
traduction ou d'extraduction français ${ }^{9}$ au sein desquels ce sont généralement les éditeurs ou les traducteurs qui choisissent les œuvres à traduire, une liste est ici soumise aux éditeurs et/ou aux traducteurs afin de pouvoir bénéficier de la subvention. Même si des aménagements sont sans doute possibles, l'une des caractéristiques de ce dispositif est de mettre en place une forme de politique littéraire (au sens de politique linguistique) prise en charge à la fois par l'OPLB et le Conseil régional de Bretagne. La littérature de langue bretonne est donc un espace très hétéronome en raison de son enjeu principal, la défense de la langue.

\section{Analyser la liste des œuvres proposées à la (re)traduction}

Après une première liste de 92 œuvres établie en décembre 2013, celle-ci s'est étoffée au fil des années et des traductions réalisées jusqu'à atteindre 129 œuvres et 122 auteurs en décembre 2018 (liste toujours en vigueur au $1^{\text {er }}$ mars 2021), dont 33 ont d'ores et déjà été traduites ; on y trouve 18 œuvres en français, dont 6 traduites ${ }^{10}$. Cette sélection et les ajouts successifs ont été établis par la commission littéraire de I'OPLB selon plusieurs critères: reconnaissance du caractère classique des ouvrages ; représentativité des différents genres, de la diversité géographique et linguistique, ainsi que du rapport auteurs-rices; un choix également établi en fonction de ce qui a déjà été traduit en langue bretonne.

7. Literatura Unibertsala, voir eizie.eus/fr/argitalpenak/literatura_unibertsala, consulté le ${ }^{\text {er }}$ mars 2021.

8. Voir par exemple «La promotion internationale du livre français », www.diplomatie.gouv.fr, et «Aide aux éditeurs pour la traduction d'ouvrages français en langues étrangères », centrenationaldulivre.fr, consultés le $1^{\mathrm{er}}$ mars 2021.

9. Le terme « intraduction » réfère aux ouvrages traduits dans la langue du pays d'édition à partir d'un texte écrit dans une langue étrangère. Par « extraduction », on désigne les livres « exportés » d'un pays via la traduction.

10. "Les ouvrages en français sont souvent des classiques étudiés au collège ou au lycée : Le Misanthrope, L'Étranger de Camus... ", entretien avec Olier Ar Mogn, responsable scientifique de l'OPLB, réalisé par mail en février et mars 2021. 
La comparaison de cette liste avec celle établie en 2002 par le Cercle norvégien du livre $^{11}(C N L)$ - dont on peut considérer qu'il s'agit d'une liste de référence -, permet de faire ressortir certaines singularités. Dans ces deux listes, les auteurs sont très largement plus nombreux que les autrices ${ }^{12}$; un constat qui vaut également pour les auteurs d'ouvrages de langue française. Par contre, les ouvrages en anglais constituent presque la moitié de la liste OPLB (dont pas moins de 8 auteurs issus de pays celtiques, Écossais, Irlandais ou Gallois), contre un tiers des ouvrages de la liste $\mathrm{CNL}^{13}$. De même, plus des trois-quarts des œuvres de la liste OPLB datent du XX $x^{\mathrm{e}}$ siècle $\left(93,2 \%\right.$ avec celles $d u X X l^{e}$ siècle ; la répartition étant similaire pour les ouvrages en langue française), contre $46 \%$ de la liste norvégienne ${ }^{14}$. De même que dans la liste norvégienne, le principal genre littéraire de la liste OPLB est le roman (dont un grand nombre de romans de science-fiction, policier et pour la jeunesse), suivi de la nouvelle $^{15}$, dans les mêmes proportions pour les ouvrages en français. L'œuvre-type de la liste OPLB serait donc un roman du $x x^{e}$ siècle écrit par un écrivain anglophone. Par ailleurs, cette surreprésentation des ouvrages policiers, de science-fiction comme des bandes dessinées dans la liste OPLB diffère de ce genre de listes de 'classiques' qui mettent généralement en avant les œuvres appartenant au pôle de production restreinte ${ }^{16}$. Enfin, toujours en croisant la liste OPLB avec la liste CNL, on ne retrouve que 24 auteurs et 9 œuvres en commun (2 auteurs et une œuvre pour les ouvrages en français); il nous semble que la liste OPLB comprend un nombre relativement réduit d'auteurs parmi les plus légitimes et, quand ceux-ci s'y trouvent, les œuvres à traduire ne sont que rarement les plus (re-)connues.

Il y a quelques classiques du XX $x^{\mathrm{e}}$ siècle (Camus, Giono et Yourcenar) parmi les ouvrages en français de la liste OPLB, mais aucun auteur des XVIII ${ }^{\mathrm{e}}$ et $\mathrm{XIX} \mathrm{X}^{\mathrm{e}}$ siècles, qui comptent pourtant un grand nombre d'écrivains inscrits au panthéon de la littérature mondiale : notamment, Diderot, Flaubert, Balzac ou encore Stendhal, présents dans la liste CNL. On retrouve, a contrario, dans la liste OPLB, des auteurs qui traitent de la Bretagne comme d'un objet littéraire (Henri Queffélec, Yves Elleouët, François Bourgeon, Julien Gracq, Kris), ainsi que des œuvres de littérature dite francophone.

Depuis 2013, 33 ouvrages de cette liste ont été traduits : $75 \%$ d'entre eux sont des romans - ce qui est normal, $70 \%$ des ouvrages de la liste sont des romans -, très majoritairement du $x x^{\mathrm{e}}$ siècle, dont une proportion relativement grande de romans pour la jeunesse. La majorité de ces ouvrages ont été intraduits de l'anglais $(57,6 \%)$, du français $(18,2 \%)$ et de I'italien (12,1\%). En français, ce sont trois romans, une nouvelle, une pièce de théâtre et une

11. Site du Cercle norvégien du livre, greaterbooks.com/norway, consulté le $1^{\mathrm{er}}$ mars 2021.

12. Liste OPLB : hommes, $82,8 \%$; femmes, 17,2 . Liste CNL : hommes, $85 \%$; femmes, $10 \%$; anonymes, $10 \%$.

13. Liste OPLB : anglais, $49,6 \%$; français, $14 \%$; espagnol, $13,2 \%$; allemand, $7 \%$; italien, 3,9 \%. Liste CNL : anglais, $29 \%$; français, $12 \%$; allemand, $10 \%$; russe, $9 \%$; espagnol, $6 \%$.

14. Liste OPLB : $X X^{\mathrm{e}}$ siècle, $77,5 \%, X X I^{\mathrm{e}}$ siècle, $14,7 \%$; XIXe siècle, $4,7 \% ; X V I I^{\mathrm{e}}$ siècle, 3,1 \%. Liste CNL : $X X^{\mathrm{e}}$ siècle, $46 \%$; XIXe siècle, $26 \%$; Antiquité, $11 \%$; Moyen Âge, $8 \%$, XVIe et XVII siècles, $6 \%$ et XVIII ${ }^{e}$ siècle, $3 \%$.

15. Liste OPLB : roman, $69,8 \%$ (dont roman de science-fiction, $9,3 \%$; roman policier, $7 \%$; roman jeunesse, 5,4 $\%)$; nouvelle, $11,6 \%$. Liste CNL : roman, $66 \%$; poésie, $16 \%$; théâtre, $8 \%$; nouvelle, $6 \%$.

16. Dominique Faria, "La traduction, instrument de légitimité littéraire », Carnets, Deuxième série, $n^{\circ}$ 9, 2017, p. 2. 
bande dessinée qui ont été traduits, dont la moitié date du $x x^{e}$ siècle. Ces 33 ouvrages ont été publiés dans les maisons d'édition Mouladurioù hor yezh $\left(14^{17}\right)$, An Alarc'h $\left(9^{18}\right)$, Al Liamm $\left(3^{19}\right)$, Keit vimp bev $\left(2^{20}\right)$, Le temps Éditeur/An Amzer $\left(2^{21}\right)$, Aber $\left(1^{22}\right)$, Barn ha skrid $\left(1^{23}\right)$, Le papier timbré $\left(1^{24}\right)$, toutes installées en Bretagne (sauf une en Loire-Atlantique) et qui publient pour la plupart d'entre elles exclusivement des ouvrages en langue bretonne. Les tirages de ces traductions sont en moyenne de 350 à 400 ouvrages et les ventes sont en moyenne de 150 à 200 exemplaires ${ }^{25}$; ce qui correspond peu ou prou aux chiffres de ventes habituels de la plupart de ces éditeurs.

\section{(Re)traduire pour gagner en capital littéraire}

La mise en place de ce dispositif de traduction/retraduction implique de nombreux acteurs: maisons d'édition, traducteurs-rices, institution régionale, OPLB et sa commission littéraire ; de toute évidence, ces dernières années la traduction a-t-elle constitué pour ces acteurs un enjeu important pour la promotion de la langue, au point de faire le choix de mettre les ouvrages en français dans la liste des ouvrages à traduire, au même titre que des œuvres étrangères. Un argument pour faire valoir ces traductions consiste à mettre en évidence la nécessité de faire du breton une langue 'comme les autres' : si Molière, Giono ou, plus encore, Jules Verne ont été traduits dans toutes les langues du monde, ou presque, il n'y aurait pas de raison qu'ils ne le soient pas en breton. Toujours selon cette logique, les traductions issues du français sont présentées comme un apport extérieur, au même titre que les autres, faisant ainsi abstraction des compétences linguistiques et culturelles de l'ensemble du lectorat du breton. Ce choix semble aussi avoir été celui de la revue littéraire Al Liamm dans les années 1960. Même si les œuvres (re)traduites du français y étaient relativement peu nombreuses par rapport aux traductions issues des autres langues, celtiques, en particulier -, des extraits d'œuvres pouvant être considérées comme des 'classiques' de la littérature française avaient trouvé place dans cette revue ; ceux-ci se caractérisaient par leur appartenance à la littérature la plus légitime du champ et/ou connaissant une forte diffusion scolaire, par exemple

17. Le Misanthrope, The Poor Mouth, Shining, Se questo è un vomo, Il Visconte dimezzato, The Call of the Wild, Breakfast at Tiffany's, El Coronel no tiene quien le escriba, Il Giorno prima della felicità, Reunion, Il Barone rampante, Regain, Il Cavaliere inesistente.

18. Histoire comique des états et empires de la lune et du soleil, Der Prozeb, The Wind in the Willows, Un recteur de l'île de Sein, The Speckled People, Himnariki og holviti, The Handmaid's Tale, The Picture of Dorian Gray, Schachnovelle.

19. No Great Mischief, The Catcher in the Rye, Amongst Women.

20. Murder on the Orient Express, The Murder of Roger Ackroyd.

21. Harry Potter and the Philosopher's Stone, Harry Potter and the Chamber of Secrets.

22. The Jungle Book.

23. L'Étranger.

24. Persepolis.

25. Si on ne prend pas en compte les deux volumes traduits de la série Harry Potter vendus à 2400 et 700 exemplaires qui constituent deux exceptions notables. 
Montaigne ${ }^{26}, \operatorname{Vian}^{27}$, Baudelaire ${ }^{28}$, Prévert ${ }^{29}$ ou encore Verlaine ${ }^{30}$. On peut constater que ces auteurs, (re)traduits ponctuellement dans Al Liamm, sont aujourd'hui absents de la liste OPLB, qui a donc été établie sur des critères différents.

La justification qui pousse à présenter les œuvres issues du français comme un apport 'comme un autre', voire comme un apport « étranger ${ }^{31}$ » semble moins faire partie des justifications des promoteurs de cet actuel dispositif, elle a en revanche constitué, par le passé, un argument simple, voire simpliste, faisant abstraction de «la nature intrinsèquement asymétrique des contacts entre les littératures ${ }^{32}$ ». De fait, la (re)traduction en breton d'œuvres issues du français avait pu être présentée par certains militants, dès la période de l'entre-deux guerres, comme une manière pour la littérature en langue bretonne de 'rattraper son retard' : «Diskiant e vefe gortoz ar skrivagnerion vrezhonek da sevel anezho o unan levrioù a-seurt gant ar re-se. Gwellañ ha berrañ hent eo hemañ : treiñ diwar yezhoù all33 » [ II serait insensé d'attendre des écrivains bretons qu'ils produisent eux-mêmes des livres de cette sorte. La meilleure route et la plus courte est de traduire à partir des autres langues].

S'il est « insensé » d'attendre que les innovations littéraires des champs dominants arrivent d'elles-mêmes dans les œuvres en langue bretonne, dès lors la (re)traduction constitue-t-elle une manière de combler un décalage temporel entre des champs fonctionnant sur des modèles sociolittéraires et sociolinguistiques très différents ${ }^{34}$. La (re)traduction revêt donc une autre dimension, elle n'est pas uniquement symbolique, c'est également une manière pour la langue de gagner en capital littéraire : il s'agit ici encore du phénomène de «traduction-accumulation » décrit par Pascale Casanova35. Ainsi, tandis que les lecteurs font l'expérience de la re-lecture ou d'un « dépaysement en trompe I'œili6 ${ }^{3}$ en lisant, en breton, des œuvres issues de patrimoine littéraire français, la littérature en langue bretonne accumule du capital littéraire. Et ce gain ne peut se faire qu'en s'appuyant sur un champ littéraire reconnu : la (re)traduction du français présente le double avantage du prestige littéraire et de la proximité culturelle immédiate, que ce soit pour les traducteurs ou pour les lecteurs. Le

26. «Prederiañ zo deskiñ mervel » [Que philosopher, c'est apprendre à mourir], Al Liamm, n 53, 1955, p. 38.

27. «An Alumetez » [Les pompiers], Al Liamm, n 143, 1970, p. 476.

28. Plusieurs poèmes de Baudelaire ont été traduits en breton entre 1955 et 2002, voir les numéros 48, 257, 266, 306, 331 de la revue Al Liamm.

29. Plusieurs poèmes de Prévert ont été traduits en breton entre 1951 et 1972, voir les numéros 28, 42, 150 de la revue Al Liamm.

30. « Huñvre bemdeiz » [Mon rêve familier], Al Liamm, n 48,1955, p. 83.

31. Jacques-Yves Mouton, Al Liamm (1945-1980). Contribution à la construction identitaire des néo-bretonnants d'après-guerre, thèse de doctorat, Université de Bretagne occidentale, 2009, p. 19.

32. Rainer Grutman, "L'autotraduction : de la galerie de portraits à la galaxie des langues », Glottopol, $n^{\circ} 25$, 2015, p. 14-30.

33. Mordiern cité et traduit par Erwan Hupel, Gwalarn histoire d'un mouvement littéraire en Bretagne, thèse de doctorat, Université Rennes 2, 2010, p. 152.

34. Nelly Blanchard et Mannaig Thomas, "Qu'est-ce qu'une périphérie littéraire ? ", dans Nelly Blanchard et Mannaig Thomas (dir.), Des littératures périphériques, Rennes, Presses universitaires de Rennes, 2014, p. 1123.

35. Pascale Casanova, "Consécration et accumulation... », op. cit., p. 10.

36. Erwan Hupel, « Le cœur et l'esprit : déchirements et stratégies d'autotraduction chez quelques auteurs bretons. ", Glottopol, n²5, 2015, p. 124-135. 
gain en capital littéraire est toutefois à nuancer pour le dispositif d'aides à la traduction décrit ci-dessus : en principe, le gain se fait essentiellement par la traduction d'œuvres issues du champ restreint, à savoir les œuvres les plus 'exigeantes'. Si cela a pu être le cas dans les décennies précédentes (cf. les traductions de Baudelaire, Montaigne ou Verlaine dans la revue Al Liamm notamment), dans la liste OPLB, ni Proust, ni Gide, ni Beckett, ni Bonnefoyet, plus globalement, absence du genre poétique -, ne sont proposés à la (re)traduction. Si un gain en capital littéraire est recherché, un compromis semble également nécessaire entre la sélection d'œuvres exigeantes et le souhait que ces œuvres puissent avoir le plus de chances d'être diffusées auprès du public, en particulier du public des jeunes néolocuteurs ${ }^{37}$.

Cette inégalité entre champs littéraires transparaît également de manière plus structurelle. Contrairement aux États qui financent souvent en priorité la traduction de leur littérature dans d'autres langues, ici, seule l'intraduction fait partie du dispositif, ce qui s'explique sans doute par l'origine du financement : il a été mis en place par le Service des langues du Conseil régional de Bretagne; il s'agit donc, avant tout, d'un dispositif de promotion de la langue bretonne, et non de diffusion de la littérature bretonne.

\section{(Re)traduire pour accumuler du capital linguistique}

Ces (re)traductions pourraient également constituer une manière de 'former' la langue bretonne, autrement dit, d'accumuler du capital linguistique. Tout d'abord, parce que le dispositif implique, pour les maisons d'édition, de se plier à une procédure de vérification de la correction linguistique des textes proposés et ceci après validation de plusieurs critères: maîtrise de la langue source, correction grammaticale et syntaxique de la traduction, respect du niveau de langue, style de la traduction et sa fidélité dans la langue-cible. Il est évident que des problématiques relevant à la fois de la traductologie et de la sociolinguistique peuvent se poser aux (re)traducteurs qui doivent rendre en breton des mots, mais également des concepts, pas forcément présents dans la langue bretonne. L'analyse que propose Zohar Shavit du phénomène de la traduction de classiques internationaux en hébreu dans la perspective de la constitution d'une littérature hébraïque est en cela comparable sous bien des aspects :

Quiconque traduisait en hébreu prenait part à la construction de la langue hébraïque. Nous nous penchions, jour et nuit, sur un mot et nous devisions ensemble sur son sens, butant sur des concepts qui sont si simples et faciles dans une autre langue, et cependant si durs et incompréhensibles en hébreu ${ }^{38}$.

Comme pour d'autres domaines de la vie sociale (traductions administratives diverses, signalétique routière $39 . .$.$) , cette problématique est essentiellement prise en charge par l'OPLB$

37. Entretien avec Ronan Le Louarn, chef du Service des langues de Bretagne au Conseil régional de Bretagne, réalisé par mail en février 2021.

38. Samuel Joseph-Agnon cité dans Zohar Shavit, «Fabriquer une culture nationale. Le rôle des traductions dans la constitution de la littérature hébraïque ", Actes de la recherche en sciences sociales, $\mathrm{n}^{\circ}$ 144, 2002, p. 27.

39. Nelly Blanchard, Ronan Calvez et Mannaig Thomas, «Signe et sens en balance. Le breton affiché dans la ville de Brest ", International Journal of the Sociology of Language, n 223, 2013, p. 137-152. 
dans le cadre d'une politique de normalisation linguistique dans laquelle s'inscrit, en toute logique, ce programme de traduction littéraire $4^{\circ}$. À court terme, l'un des effets recherchés par ce dispositif est également celui de la professionnalisation des traducteurs et des auteurs. Les livres traduits l'ont été par 15 traducteurs entre 30 et 81 ans (59 ans en moyenne), dont la plupart travaillent ou ont travaillé dans l'enseignement : aucun d'entre eux n'est traducteur professionnel et ce profil correspond peu ou prou à celui des auteurs en langue bretonne. La possibilité d'être rémunérés leur permet d'une part, de travailler dans de bonnes conditions et, d'autre part, d'accepter « de travailler sur proposition des éditeurs et non plus seulement par goût personnel pour telle ou telle œuvre ou auteur ${ }^{41} »$. En effet, dans un espace littéraire basé jusque-là presqu'exclusivement sur le volontariat et le bénévolat, la mise en place d'une politique éditoriale et d'un programme de traduction ambitieux n'est pas évidente pour des maisons d'édition très dépendantes de l'engagement militant des auteurs. De plus, certain.e.s traducteurs.rices sont également auteurs-rices, comme le précise Ronan Le Louarn : « les retours des traducteurs sont positifs : ce sont très souvent des auteurs par ailleurs et la rémunération de leur travail de traduction tranche avec les très faibles droits d'auteurs qu'ils peuvent toucher sur leurs œuvres originales, compte tenu du tirage moyen des œuvres en langue bretonne. » Au vu du marché économique que représente la littérature en langue bretonne: « une traduction professionnelle rémunérée serait impossible sans l'aide régionale ${ }^{42}$ ».

Le gain en capital linguistique passe par une procédure de vérification qui ne semble pas exister aussi formellement dans les autres dispositifs d'aide à la publication en langue bretonne, peut-être parce que, pour des 'classiques' ou des 'chefs-d'œuvres', il y a nécessité de faire preuve d'une exigence toute particulière. De fait, « la question n'est donc pas tant le fait de pouvoir lire telle ou telle œuvre dans la langue originale, mais de souhaiter en lire une re-création en langue bretonne. Re-création qui vient enrichir l'imaginaire de langue bretonne 43 ".

Le propre des 'classiques' étant, par définition, d'être destinés à se perpétuer, le dispositif se place dans une perspective de longue durée. Cette stratégie volontariste de gain en capital linguistique vise sans doute à améliorer progressivement la production des œuvres en langue bretonne, à la fois dans leur dimension littéraire - «l'imaginaire ${ }^{44} 》-$, mais également linguistique - «permettre à la langue bretonne d'aborder des lieux et des époques différentes 45 ». L'évaluation concrète de l'apport linguistique des retraductions, en termes lexicaux, par exemple, n'est pas directement l'objet de cet article. Pour chaque œuvre, des problématiques spécifiques en termes de vocabulaire, par exemple, peuvent se poser aux traducteurs : rendre la nature provençale de Regain / Ar Gwim, faire usage d'un vocabulaire à

\footnotetext{
40. Voir par exemple les différentes ressources linguistiques présentées sur le site de l'Office public de la langue bretonne, www.fr.brezhoneg.bzh/35-outils-linguistiques, consulté le ${ }^{\text {er }}$ mars 2021.

41. Entretien avec Ronan Le Louarn.

42. Ibid. Au $1^{\text {er }}$ mars 2021, le coût global du dispositif s'élève à 108 482,6o euros.

43. Entretien avec Olier Ar Mogn, responsable scientifique de I'OPLB, réalisé par mail en février et mars 2021.

44. Xavier Darcos cité dans Dominique Faria, "La traduction... », op. cit., p. 2.

45. Entretien avec Olier Ar Mogn.
} 
connotation archaïque permettant de rendre au mieux une œuvre telle que Le Misanthrope / An Dengasaour, par exemple. Ces choix peuvent constituer un gain en capital linguistique s'accumulant au fil des retraductions, même si une évaluation concrète de la diffusion de ces choix, auprès du lectorat notamment, serait difficile à évaluer avec précision.

\section{(Re)traduire pour former le lectorat}

Au-delà d'un intérêt strictement symbolique, voire 'de façade', les (re)traductions doivent donc être interprétées dans la perspective d'une amélioration globale de la production littéraire en breton, à la fois en amont (gain de capital littéraire, formation de professionnels et extension des domaines d'usage de la langue), mais également en aval. En effet, en bout de chaîne, ce dispositif pourrait également avoir pour ambition, à long terme, de former un lectorat, c'est-à-dire d'agir sur la réception des œuvres. La question de la lecture en breton constitue une illustration de la situation sociolinguistique de la langue et sa pratique est fortement corrélée à la répartition du lectorat en termes de classe d'âge. Schématiquement, il y a, d'une part, les locuteurs âgés (près de $80 \%$ des locuteurs du breton ont plus de 60 ans ${ }^{46}$ ) ayant une pratique paritaire de la langue, qu'ils ne lisent pas ou très peu et d'autre part, la catégorie des locuteurs les plus jeunes, entre 15 et 24 ans, qui ont une pratique presque exclusivement scolaire de la langue, qu'ils sont en mesure de lire et d'écrire. Ces chiffres très contrastés sont une illustration supplémentaire de la pratique tout aussi contrastée de la langue bretonne, mais également de la complexité des rapports des deux catégories de locuteurs à la lecture. Pour la première catégorie de locuteurs, la lecture en breton est soit inexistante, soit difficile. Ainsi, à la question "Savez-vous lire le breton ? ", $39 \%$ des locuteurs, déclarant par ailleurs parler bien ou très bien, répondent « pas du tout » et $33 \%$ répondent "un peu », soit une pratique inexistante ou très faible pour $72 \%$ des locuteurs ${ }^{47}$.

Les nuances dans les réponses aux questions sur les capacités de lecture et d'écriture en breton expriment d'ailleurs bien la complexité du rapport à l'écrit pour ces générations de locuteurs les plus âgés : alors que l'on attendrait une réponse par oui ou non, savoir lire/écrire ou ne pas savoir lire/écrire, on constate une déclinaison allant de «non, pas du tout ", à « un peu ", "assez bien " ou «très bien ». À l'autre extrême de la courbe d'âge, les locuteurs de moins de 24 ans affirment quant à eux savoir lire le breton à $98 \%$. Si ce dernier chiffre témoigne d'une compétence réelle acquise dans le cadre scolaire, il souligne également que, par l'enseignement reçu en breton, ces locuteurs s'autorisent à se considérer comme des lecteurs-rices légitimes. Pour autant, la pratique de la lecture en breton de cette génération reste à interroger ${ }^{48}$ car de nombreux acteurs du monde de l'édition et de l'enseignement en breton

46. Pascale Wakeford et Fañch Broudic, Les langues de Bretagne. Enquête sociolinguistique, octobre 2018. À consulter sur www.bretagne.bzh.

47. Yves Le Berre et Jean Le Dû, «Parité et disparité : sphère publique et sphère privée de la parole », La Bretagne linguistique, $\mathrm{n}^{\circ} 10,1996, \mathrm{p} .7-25$.

48. Eve Rouxel, «Bilinguisme scolaire et médiation littéraire dans les salons et festivals du livre jeunesse : le cas de la littérature de jeunesse en langue bretonne ", RELIEF - Revue électronique de littérature française, vol. 14, $n^{\circ} 2$, 2020, p. $73-86$. 
s'accordent sur le décalage entre les compétences linguistiques et de lecture des jeunes locuteurs et les ouvrages qui retiendraient leur intérêt; un décalage d'autant plus saillant par comparaison avec ce que ces locuteurs sont en mesure de lire en français, à la fois en raison de la diversité de l'offre, mais également de leurs compétences linguistiques.

La question de la (re)traduction mérite donc d'être replacée dans cette perspective, les deux grandes catégories de locuteurs du breton pouvant rencontrer, pour des raisons différentes, un rapport compliqué à la lecture. La (re)traduction peut, dès lors, être perçue comme une manière $d^{\prime} y$ remédier dans la mesure où elle permet de toucher un lectorat qui aura, par une lecture ou une connaissance préalable de l'œuvre en français, facilité son accès à l'œuvre (re)traduite en breton. C'est, en ce sens notamment que la (re)traduction peut être considérée comme une manière de former un lectorat brittophone ${ }^{49}$ et de l'accompagner dans une démarche de lecture en breton par la redécouverte d'œuvres déjà connues, déjà lues, voire déjà étudiées par les néolocuteurs dans le cadre de cours de français notamment. Ceci vaut d'ailleurs également pour des œuvres en langue étrangère découvertes une première fois en français et relues en breton ensuite : l'exemple de la traduction d'une partie de la série des Harry Potter en breton illustre la volonté de proposer à la jeunesse brittophone de relire le plus grand succès littéraire mondial des dernières décennies. Ceci rappelle, à nouveau, la description que Zohar Shavit fait du processus de constitution d'une littérature moderne en hébreu, qui est également passée par l'usage de la traduction :

Le but était bien de créer un lectorat en œuvrant à l'élaboration d'une littérature en hébreu, non de produire une littérature nationale pour un public de lecteurs déjà constitués. [...] La littérature traduite était destinée à un public qui maîtrisait plusieurs langues et dont l'hébreu n'était pas la langue maternelle. Elle essayait donc de gagner un public qui, spontanément, préférait lire des œuvres de littérature occidentale dans sa langue maternelle ou dans une langue qu'ils maîtrisaient plutôt que dans une langue hébraïque qu'il venait tout juste d'acquérir ${ }^{50}$.

L'enjeu des (re)traductions en breton nous semble être tout à fait comparable : faire en sorte que les locuteurs brittophones fassent le choix de lire des œuvres, non plus uniquement dans leur langue maternelle, le français, mais dans une langue qu'ils apprennent, faisant de cette langue non plus uniquement un objet d'apprentissage, mais progressivement l'objet d'une pratique culturelle. Comme pour l'hébreu, certains discours et enquêtes ${ }^{51}$ ont montré que les brittophones affirmaient ne pas lire en breton parce qu'ils ne trouvaient pas dans cette langue une offre suffisamment diversifiée : «si le jeune lecteur semble être repu des "plats faits maison" et souhaite goûter aux "mets du monde entier" et que le créateur en est réduit, pour un certain temps, à endosser le rôle du serviteur chargé d'apporter les plats, cela n'est pas si grave $^{52}$ ». Cette métaphore culinaire nous semble correspondre à la stratégie à long terme

49. Ronan Calvez, "Ce que parler du breton veut dire ", Ethnologie française, vol. 42, n 4, 2012, p. $647-655$.

50. Zohar Shavit, «Fabriquer une culture nationale... », op. cit., p. 23.

51. Eve Rouxel, «Bilinguisme scolaire... », op. cit.

52. Asher Barash cité par Zohar Shavit, « Fabriquer une culture nationale... », op. cit., p. 29 
mise en place par l'institution régionale bretonne pour qui la proposition d'ouvrages déjà connus en français, voire en anglais, pourrait constituer, pour certains néolocuteurs, la première marche vers une pratique de lecture en breton qui serait plus spontanée que celle découlant de l'injonction scolaire.

Pour reprendre la formule d'Alain Viala, la traduction et la (re)traduction d'œuvres littéraires en breton est donc à penser dans «I'histoire », en l'occurrence, dans le contexte actuel de la politique de promotion de la langue bretonne, focalisée sur la pratique des néolocuteurs. Ces derniers sont, certes, les moins nombreux, mais les plus jeunes, et leur pratique linguistique pourrait, selon cette stratégie au long cours, être plus facilement amenée à évoluer. L'apprentissage de la langue par les néolocuteurs dans le cadre scolaire ou de formations longues pour adultes pourrait, de ce point de vue, permettre à la fois l'extension d'une pratique linguistique, mais aussi une évolution des représentations et des discours sur la langue. Parmi les actions complémentaires qui pourraient être mobilisées dans le répertoire militant, la question est ici celle de l'extension des domaines de la pratique individuelle, au-delà d'un apprentissage exclusivement scolaire disparaissant une fois le cursus accompli : il s'agirait, comme le précise Olier Ar Mogn dans un article du journal Ouest France « de produire des locuteurs ${ }^{53}$ ». Et le modèle de la littérature hébraïque mériterait ici encore d'être convoqué : «On supposait qu'après s'être constitué d'une manière initialement artificielle, le public se développerait et se transformerait en un lectorat organique et "naturel", doté d'une assise réelle et solide qui profiterait, alors, à la création artistique en Eretz Israel54. "

\section{Bibliographie}

BLANCHARD Nelly, CALVEZ Ronan et Thomas Mannaig, "Signe et sens en balance. Le breton affiché dans la ville de Brest ", International Journal of the Sociology of Language, n²23, 2013, p. 137-152. doi.org/10.1515/ijsl2013-0049

BLANCHARD Nelly et THOMAS Mannaig, " Qu'est-ce qu'une périphérie littéraire? ", dans Nelly Blanchard et Mannaig Thomas (dir.), Des littératures périphériques, Rennes, Presses universitaires de Rennes, 2014, p. 11-23.

CALVEZ Ronan, "Ce que parler du breton veut dire ", Ethnologie française, vol. 42, n 4, 2012, p. 647-655.

CASANOVA Pascale, "Consécration et accumulation de capital littéraire. La traduction comme un échange inégal ", Actes de la recherche en sciences sociales, n 144, 2002, p. 7-20. doi.org/10.3406/arss.2002.2804

FARIA Dominique, «La traduction, instrument de légitimité littéraire ", Carnets, Deuxième série, nº $9,2017$. doi.org/10.4000/carnets. 2083

GRUTMAN Rainer, "L'autotraduction : de la galerie de portraits à la galaxie des langues », Glottopol, $n^{\circ} 25,2015$, p. 14-30. À consulter sur glottopol.univ-rouen.fr

HUPEL Erwan, Gwalarn histoire d'un mouvement littéraire en Bretagne, thèse de doctorat, Université Rennes 2, 2010.

- «Le cœur et l'esprit : déchirements et stratégies d'autotraduction chez quelques auteurs bretons. », Glottopol, 2015, n²5, p. 124-135. À consulter sur glottopol.univ-rouen.fr

53. Emmanuel Cadieu, «Breton : traduire des livres et faire avancer la langue », entretien avec Olier Ar Mogn, Ouest France, www. ouest-france.fr, 2 mars 2020, consulté le $1^{\mathrm{er}}$ mars 2021.

54. Zohar Shavit, «Fabriquer une culture nationale... », op. cit., p. 23. 
Le BerRe Yves et Le Du Jean, "Parité et disparité : sphère publique et sphère privée de la parole », La Bretagne linguistique, $\mathrm{n}^{\circ} 10,1996$, p. 7-25.

Mouton Jacques-Yves, Al Liamm (1945-1980). Contribution à la construction identitaire des néo-bretonnants d'après-guerre, thèse de doctorat, Université de Bretagne occidentale, 2009.

ROUXEL Eve, « Bilinguisme scolaire et médiation littéraire dans les salons et festivals du livre jeunesse : le cas de la littérature de jeunesse en langue bretonne ", RELIEF - Revue électronique de littérature française, vol. 14 $\mathrm{n}^{\circ}$ 2, 2020, p. 73-86. doi.org/10.18352/relief.1092

SHAVIT Zohar, «Fabriquer une culture nationale. Le rôle des traductions dans la constitution de la littérature hébraïque ", Actes de la recherche en sciences sociales, n 144, 2002, p. 21-32. doi.org/10.3917/arss.144.0021

VIALA Alain, "Qu'est-ce qu'un classique? ", Littératures classiques, n 19, 1993, p. 11-31. doi.org/10.3406/licla. 1993.1737

VINCENT GERARD Armelle et PONCET Julie, Les Français et la lecture-2019. CNL-Ipsos, 2019. À consulter sur www. ipsos.com 